\title{
Awake, percutaneous, bedside extracorporeal membrane oxygenator arterial cannula exchange
}

\author{
Mohamed Abdullah, MD, PhD, Aakash Shah, MD, Ronson J. Madathil, MD, and \\ David J. Kaczorowski, MD
}

Video clip is available online.

Although uncommon, fracture of an extracorporeal membrane oxygenator (ECMO) cannula with consequent bleeding requires immediate management. We describe a safe technique that can be employed at the bedside without the use of general anesthesia or surgical incisions for a venoarterial ECMO peripheral cannula exchange.

\section{CASE REPORT}

A 54-year-old man presented with cardiogenic shock due to decompensated nonischemic cardiomyopathy. He was placed on a venoarterial ECMO, through cannulation of the right common femoral artery and vein. A distal perfusion catheter was placed in the superficial femoral artery (SFA) and connected to the arterial cannula. The patient was stabilized. A small fracture at the side port of the arterial cannula was noted by the nursing staff in the intensive care unit, as there was slow continuous blood emanating from the cannula. The fracture was most likely caused by mechanical strain, as the cannula was secured to the skin at this site. Topical agents were applied to temporize the bleeding. However, cannula exchange was indicated. To avoid transfer of the patient and any delay while waiting for an operating room (OR), cannula exchange was performed at bedside in the intensive care unit, using light sedation and local anesthesia. Bilateral groins, the cannulas,

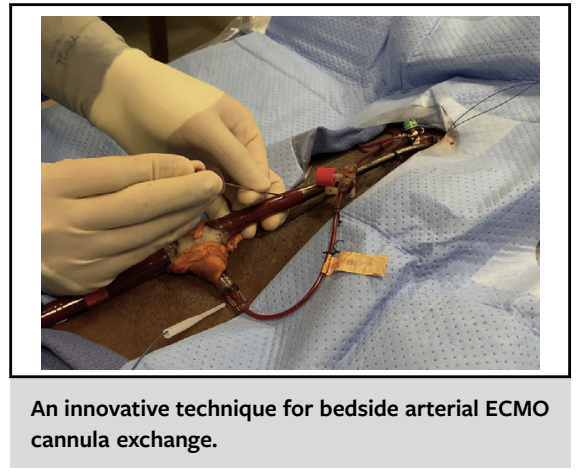

\begin{abstract}
CENTRAL MESSAGE
We report a safe and efficient technique for arterial ECMO cannula exchange in an awake patient that can be performed at the bedside.
\end{abstract}

See Commentaries on pages 86,88 , and 90 .

and the adjacent parts of tubing were prepped and draped. Heparin (40 IU/kg) was administered. ECMO was gradually weaned to a flow of $1 \mathrm{~L} / \mathrm{min}$. The cannula was accessed with an 18-G needle (Figure 1). The non-wired portion of the cannula was chosen to allow easy insertion of the needle at an angle that allowed subsequent introduction and advancement of the 0.035-inch Amplatz stiff wire toward the patient's descending aorta, as shown in the Video 1. ECMO flow was discontinued. After clamping the cannula proximal and distal to the side port, we disconnected the SFA circuit, and the tubing was cut. The cannula was then removed, while wire access was maintained throughout. Manual pressure was applied to achieve hemostasis. A new 17-French cannula was advanced percutaneously after threading the distal end of the wire into the new cannula's obturator. The cannula obturator and the wire were removed, and the cannula was clamped. The SFA circuit was then reattached. The cannula was connected to the circuit. The proximal clamp was removed, and the cannula was allowed to back bleed through the SFA circuit. The other clamp was subsequently removed. ECMO flow was reinitiated. Although hard to quantitate, we estimate that blood loss was likely less than $300 \mathrm{~mL}$. One unit of 


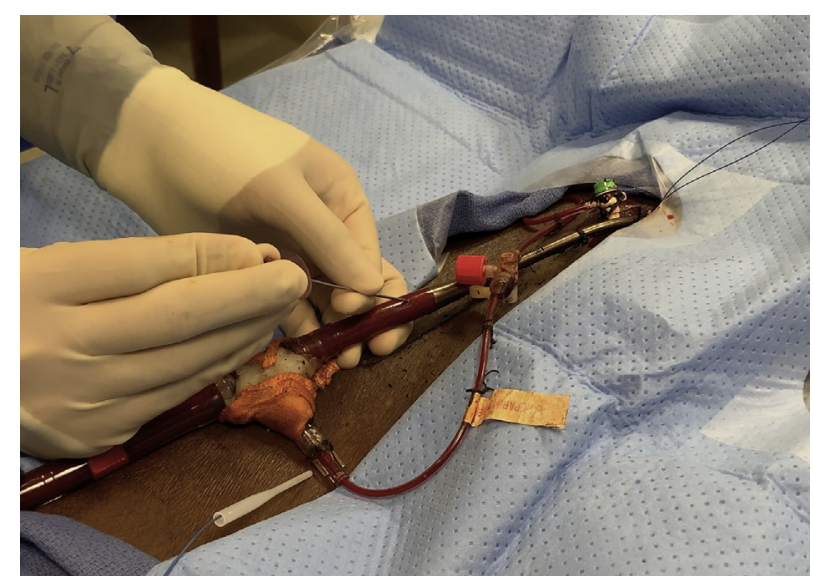

FIGURE 1. Bedside arterial ECMO cannula exchange; the cannula is accessed with an $18-\mathrm{G}$ needle, through which a stiff wire was introduced, and the cannula is exchanged over the wire.

red blood cells was transfused. The patient remained hemodynamically stable throughout and after the procedure. The patient was later decannulated successfully after transition to a left ventricular assist device. The patient provided informed consent for the publication of the study data.

\section{DISCUSSION}

Operative ECMO cannula exchange may not be feasible in emergency situations. Moreover, it requires the availability of surgical and anesthesia teams and also carries the additional risk of patient transfer and surgical incision complications. An alternative strategy is desirable. Our technique provides an efficient method of cannula exchange and avoids the need for patient transfer, general anesthesia, and surgical support staff. We also observed minimal bleeding and only a brief interruption of ECMO flow in this case, which is optimal for patients requiring circulatory support. Possible complications of this technique include bleeding, damage or dissection of the vessel, or failure to advance the new cannula. It is possible for the wire to inadvertently pass through a side hole in the tip of the cannula as it is being advanced; if there is resistance to advancing the wire, caution should be taken.

If bedside cannula exchange is to be attempted, it is important to consider backup strategies should difficulty arise. Alternative courses of action depend upon the precise issue encountered. If ECMO flow could not be reestablished, insertion of arterial cannula percutaneously in the opposite side could be performed. If there is an arterial complication on the side of the damaged cannula, the

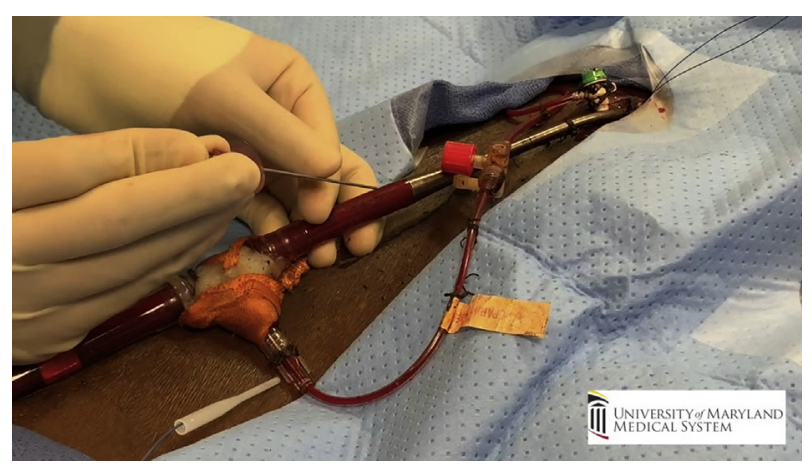

VIDEO 1. Demonstration of technique for puncturing and wiring of ECMO cannula for safe exchange. Video available at: https://www.jtcvs. org/article/S2666-2507(20)30169-3/fulltext.

patient could be transferred to the OR for surgical exposure of the vessel and exchange of the cannula or repair of the vessel. In this case, if an OR is not immediately available, manual compression should be continued to control bleeding until an OR becomes available.

Our usual strategy for cannula removal was employed in this case. Our strategy is to surgically perform a limited cutdown over the femoral artery and place a purse string suture around the arterial puncture site, then remove the cannula in a controlled manner and tie the purse string suture down. The pulse is then checked proximal and distal to the cannulation site. If pulses are absent, the artery is dissected out proximally and distally for control. The cannulation site is explored. A thrombectomy and reconstruction is then performed as necessary. The distal perfusion catheter is removed percutaneously.

A similar technique for circuit access was previously described for over-the-wire cannula removal and accesssite closure. ${ }^{1}$ While the authors hypothesized that cannula exchange might also be performed in this manner, to our knowledge, no publication has previously reported successful use of this technique for peripheral ECMO cannula exchange.

In conclusion, this technique provides a feasible and efficient approach for a controlled peripheral ECMO cannula exchange. Technology that enables endovascular access to ECMO circuits will facilitate this technique, as well as other interventions through the circuit itself.

\section{Reference}

1. Heller S, Kovárník T, Linhart A, Belohlávek J. Puncture and wiring of extracorporeal circuit for cannula safe removal or exchange. Perfussion. 2016;31: 604-7. 